\title{
In vitro irradiation station for broad beam radiobiological experiments
}

\author{
A.-C. Wéra ${ }^{1}$, H. Riquier ${ }^{2}$, A.-C. Heuskin ${ }^{1}$, C. Michiels ${ }^{2}$, S. Lucas ${ }^{1}$ \\ ${ }^{1}$ NAmur Research Institute for LIfe Sciences (NARILIS), Research Centre for the Physics of \\ Matter and Radiation (PMR), University of Namur-FUNDP, \\ Corresponding author: anne-catherine.wera@fundp.ac.be \\ ${ }^{2}$ NAmur Research Institute for LIfe Sciences (NARILIS), Unité de Recherche de Biologie \\ Cellulaire (URBC), University of Namur-FUNDP \\ Rue de Bruxelles, 61, B-5000 Namur, Belgium
}

\section{$\underline{\text { Abstract }}$}

The study of the interaction of charged particles with living matter is of prime importance to the fields of radiotherapy, radioprotection and space radiobiology.

Particle accelerators and their associated equipment are proven to be helpful tools in performing basic science in all of these fields. Indeed, they can accelerate virtually any ions to a given energy and flux and let them interact with living matter either in vivo or in vitro.

In this context, the University of Namur has developed a broad beam in vitro irradiation station for use in radiobiological experiments. Cells are handled in GLP conditions and can be irradiated at various fluxes with ions ranging from hydrogen to carbon. The station is mounted on a $2 \mathrm{MV}$ Tandem accelerator, and the energy range can be set up in the LET ranges that are useful for radiobiological experiments.

This paper describes the current status of the hardware that has been developed, and presents results related to its performance in term of dose-rate, energy range and beam uniformity for 
protons, alpha particles and carbon ions. The results of clonogenic assays of A549 lung adenocarcinoma cells irradiated with protons and alpha particles are also presented and compared with literature. 


\section{$\underline{\text { Introduction }}$}

Since 1946, with the proposal of R. R. Wilson [1], the use of charged particles in radiobiology has encountered an increasing interest. The development of cancer treatments using particle accelerators has continued to this day. In 2005, U. Amaldi found that more than $40 \%$ of the 17,500 existing particle accelerators were used for radiotherapy, with about 40,000 and 2,200 patients treated with proton beams and carbon ions respectively [2]. At the same time, fundamental research performed with particle accelerators was yielding useful information for medical applications as well as for radioprotection and space radiobiology [3-8]. The strength of a particle accelerator lies in its wide range of available ions for which energies and flux can be greatly varied. There are two configurations: microbeams and broad beams. On the one hand, micro/nano-beams allow the irradiation of a selected cell, or a portion of a cell, with a precise number of ions [9-16]. However, microbeam installation requires tedious developments for cell recognition, alignment and beam scanning. On the other hand, broad beams are easier to implement [17-22]. Furthermore, this system also allows simultaneous treatment of thousands of cells within minutes. Therefore, with broad beams, additional stresses like temperature effects or absence of culture medium during irradiation can be minimized. Nevertheless, as is the case for patient irradiation, not all the cells can be targeted because of the Poisson distribution of the beam.

In 2007, the LARN laboratory at the University of Namur started to develop an inexpensive broad beam in vitro irradiation station. First results were published in 2008 regarding proton beam characterization [23]. In this paper, we describe the hardware development for our irradiation station, as well as present results related to its performance in term of energy 
straggling, beam uniformity and monitoring for proton, alpha and carbon ions beams. The irradiation station was used to irradiate A549 non-small-cell lung cancer adenocarcinoma cells (NSCLC) with proton and alpha beams at $1 \mathrm{~Gy} / \mathrm{min}$. The survival fraction curves assessed by conventional clonogenic assays are compared to literature. Radiosensitivity parameters, as well as the inactivation cross-section, are calculated.

\section{$\underline{\text { Material and Methods }}$}

\section{Irradiation facility}

The irradiation station is placed on the $2 \mathrm{MV}$ Tandem accelerator (High Voltage Engineering Europa) available at the LARN laboratory. Thanks to its dual source, particles from Hydrogen to Uranium can be accelerated with a terminal voltage adjustable from 0.15 to $2 \mathrm{MV}$. The irradiation station is fixed at the end of the $10^{\circ}$ exit port of the switching magnet. The irradiation field is set up by defocusing the beam with the help of electrostatic and magnetic optical elements mounted along the beam line. The irradiation station is schematically presented in Figure 1 . It consists of a vacuum chamber (2) in which a removable CCD camera is mounted with a BC400 scintillator (4). The camera is used to tune the beam at a high current. The beam is extracted to air through an exit window (6) made of a $8 \mu \mathrm{m}$ Kapton foil maintained between two stainless steel cylinders (see insert of Figure 1). A collimated PIPS (Passivated Implanted Planar Silicon) detector is placed just before the irradiation head (5). This detector intercepts the left side of the beam and is used as a dose-rate monitor during the irradiation. Another PIPS detector (7) is fixed on an XY table, which is activated by a step motor (8) and which is placed just after the exit 
window. The exposed surface of this detector was limited with a collimator $(\varnothing 510 \pm 2 \mu \mathrm{m})$. This is used to adjust the dose-rate, and to assess the stability and the homogeneity of the broad beam.

\section{Dosimetry}

The dose-rate is given by:

$$
\dot{D}(\mathrm{~Gy} / \mathrm{s})=1.6 \times 10^{-9} \frac{\operatorname{LET}(\mathrm{keV} / \mu \mathrm{m}) . \Phi\left(\mathrm{part} / \mathrm{cm}^{2} . \mathrm{s}\right)}{\rho\left(\mathrm{g} / \mathrm{cm}^{3}\right)}
$$

where LET is the linear energy transfer, $\Phi$ is the flux and $\rho$ is the cell density. Therefore, to perform radiobiological studies with a broad beam, the LET and the flux need to be known and constant over the entire surface covered by the biological cells and throughout the irradiation.

As the LET is related to the beam energy, a precise energy calibration of the accelerator is necessary. The experimental detail and results of the calibration were presented in [24]. The LET value, calculated by the SRIM program [25], can be scanned from 10 to $50 \mathrm{keV} / \mu \mathrm{m}$ for proton and from 90 to $200 \mathrm{keV} / \mu \mathrm{m}$ for alpha particles with energies ranging from $4 \mathrm{MeV}$ to $800 \mathrm{keV}$ and from $6 \mathrm{MeV}$ to $3 \mathrm{MeV}$ for proton and alpha particles respectively. For carbon ions, the Kapton exit foil is replaced by a $3 \mu \mathrm{m}$ Mylar foil, and LET from 650 to $900 \mathrm{keV} / \mu \mathrm{m}$ are achievable.

The flux can be adapted to obtain a large range of dose-rates (typically from 0.1 to $10 \mathrm{~Gy} / \mathrm{min}$ ). Its stability is checked over time and over the irradiation surface. The beam uniformity is checked by moving the PIPS millimeter by millimeter to obtain a 1 x $1 \mathrm{~cm}$ map. The results presented in this paper were obtained for a fixed dose-rate of $1 \mathrm{~Gy} / \mathrm{min}$ for all ions $\left({ }^{1} \mathrm{H}^{+},{ }^{4} \mathrm{He}^{2+},{ }^{12} \mathrm{C}^{4+}\right)$. 


\section{Cell culture and irradiation}

Human A549 NSCLC cells were grown in MEM (Minimum Essential Medium) (Invitrogen, UK) containing 10\% (v:v) fetal calf serum (FCS) (Invitrogen, UK). Twenty-four hours before irradiation, 100,000 cells were seeded as a $35 \mu 1$ drop at the centre of the Kapton foil (exit window) of pre-sterilized irradiation heads (see figure 1). These heads are then closed with a plastic cap to avoid dehydration and contamination and are placed in an incubator at $37^{\circ} \mathrm{C}$ with 5\% $\mathrm{CO}_{2}$. Six hours after seeding, the drop is rinsed twice with phosphate-buffered saline $\mathrm{pH} 7.4$ (10 mM phosphate, $0.9 \% \mathrm{NaCl})(\mathrm{PBS})$ to remove non-adherent cells. The irradiation heads are then filled with culture medium and replaced in the incubator. Just before the irradiation, the culture medium is replaced by independent $\mathrm{CO}_{2}$ medium. After the irradiation, the $\mathrm{CO}_{2}$ medium is poured off and the irradiation heads are rinsed with PBS. The plastic cap is then removed, and a sterile cotton swab is used to take away the cells that may have detached from the central drop and diffused outside the irradiated field. The plastic cap is replaced on the irradiation heads for another PBS wash. Cells are detached by using trypsin, then counted and seeded in 6-well plates $\left(9.40 \mathrm{~cm}^{2}\right)$ at desired concentrations. Cells are also seeded in a separated dish at one concentration per dose and 2 hours later the cells are fixed with paraformaldehyde (PFA) $4 \%$ for 10 minutes before 3 PBS washes. The number of cells attached to the dish is counted manually under an optical microscope to precisely know the number of cells seeded for each concentration and dose. Eleven days post irradiation, the number of visible colonies (containing more than 50 cells) is counted after staining with crystal violet in $2 \%$ ethanol. The plating efficiency (PE) is determined for each dose and the survival fraction is calculated by evaluating the ratio of the PE for the irradiated cells to the PE for the control cells. At least three independent experiments are 
performed for each dose and the errors are evaluated as a standard deviation. Note that the control cells underwent exactly the same steps as the irradiated cells. In this study, the LET values were chosen to obtain the maximum relative biological effectiveness (RBE), which corresponds to $\sim 100 \mathrm{keV} / \mu \mathrm{m}$ and $\sim 25 \mathrm{keV} / \mu \mathrm{m}$ for alpha and proton respectively [20]. The dose-rate was fixed to $1 \mathrm{~Gy} / \mathrm{min}$.

\section{$\underline{\text { Results and Discussion }}$}

\section{Beam characterization}

\section{Energy spread}

In order to determine the dose-rate error, the energy straggling need to be determined at the cell entrance (i.e. after the $8 \mu \mathrm{m}$ Kapton foil). For that purpose, a calibrated PIPS detector was placed in the vacuum chamber and the energy spectra were recorded with and without the Kapton foil. The experiments were performed for proton and alpha particles for energies ranging from 0.7 to 3.8 $\mathrm{MeV}$ and from 4.5 to $5.9 \mathrm{MeV}$, respectively. The results presented in Figure 2 were obtained for the $1.2 \mathrm{MeV}$ proton beam. Without the Kapton foil, the energy spread characterized by the full width at half maximum (FWHM) was $7 \mathrm{keV}$ at $1.2 \mathrm{MeV}$ incident energy. With the Kapton foil, the FWHM increased to $25 \mathrm{keV}$ and the energy peaked at $952 \mathrm{keV}$. This energy straggling induced LET variations ( $\triangle \mathrm{LET}$ ) estimated at $2 \%$ at the cell mid-thickness, using the SRIM program. This variation is equal to $1 \%$ for the $5.3 \mathrm{MeV}$ alpha beam. The same experiments were performed for carbon ions with energies ranging from 5.4 to $10 \mathrm{MeV}$ and with a $3 \mu \mathrm{m}$ Mylar foil as the exit window. Without the Mylar foil, the FWHM at $10 \mathrm{MeV}$ was $70.7 \mathrm{keV}$; it increased to $192 \mathrm{keV}$ at $7.60 \mathrm{MeV}$ with the Mylar foil. This induced LET variation of less than $1 \%$ at both cell 
entrance and cell mid-thickness. In conclusion, the LET ( $\triangle$ LET) variations induced by the exit window and the passage through half of the cell layer are limited to less than $2 \%$ for the three beams.

\section{Dose-rate stability and online monitoring}

The beam stability was checked using a collimated PIPS detector placed in front of the incident beam. In Figure 3, we can observe the excellent stability $( \pm 2.8 \%)$ of the $5.3 \mathrm{MeV}$ alpha beam, for which the dose-rate was set to $1 \mathrm{~Gy} / \mathrm{min}$ with a LET of $100 \mathrm{keV} / \mu \mathrm{m}$ at the cell entrance. During irradiation, the dose-rate is monitored with another PIPS detector fixed on the left side of the beam. Indeed, we observed that the ratio between the number of particles detected in the centre of the beam to the one counted at the left side of the beam remains constant, even if the flux of the incident particle is modified. Data taken over more than one hour allowed to perform a linear fit of the ratio in function of time. This showed an angular coefficient lower than $510^{-6} \mathrm{~s}^{-1}$ even if the flux of incident particles detected in the centre of the beam decreased by more than $30 \%$ during this time. The reliability of this system allowed the control of the dose-rate during the irradiation.

\section{Beam spatial uniformity}

The beam homogeneity was checked before each irradiation using a collimated $(\varnothing 510 \mu \mathrm{m})$ PIPS detector mounted on an XY table. The flux of incident particles $(\Phi)$ is measured over a surface of $10 \times 10 \mathrm{~mm}$ by a one millimeter step. Figure 4 presents the results obtained for a $5.3 \mathrm{MeV}$ alpha beam at a dose-rate of $1 \mathrm{~Gy} / \mathrm{min}$. With our setup, an irradiation field of 6 x $5 \mathrm{~mm}$ with a dose-rate variation lower than $10 \%$ can be selected. That value includes the $2.8 \%$ beam current variation 
calculated above. Similar results are obtained for the proton beam. For carbon ions, a $3 \mu \mathrm{m}$ Mylar foil is used as exit window.

\section{Induced dose-rate error}

The dose-rate error $\Delta \dot{\mathrm{D}}$ during a typical experiment can be estimated according to the following formula if the density of target is $1 \mathrm{~g} / \mathrm{cm}^{3}$ :

$$
\Delta \dot{\mathrm{D}}=\frac{\partial \dot{\mathrm{D}}}{\partial \mathrm{LET}} \Delta \mathrm{LET}+\frac{\partial \dot{\mathrm{D}}}{\partial \Phi} \Delta \Phi=1.610^{-9}(\Phi \Delta \mathrm{LET}+\mathrm{LET} \Delta \Phi)
$$

where $\Delta$ LET and $\Delta \Phi$ are respectively the LET and the flux variation evaluated at the cell midthickness. The LET variations $(\triangle \mathrm{LET})$ induced by energy straggling are $0.5 \mathrm{keV} / \mu \mathrm{m}$ for the proton beam, $1 \mathrm{keV} / \mu \mathrm{m}$ for the alpha beam and $2 \mathrm{keV} / \mu \mathrm{m}$ for carbon ions. The flux variations $(\Delta \Phi)$ due to non-uniformity of the beam are $\pm 5 \%$ for proton, alpha and carbon. According to (2), $\Delta \dot{\mathrm{D}}$ is $0.07 \mathrm{~Gy} / \mathrm{min}, 0.06 \mathrm{~Gy} / \mathrm{min}$ and $0.05 \mathrm{~Gy} / \mathrm{min}$ for the $1.2 \mathrm{MeV}$ proton beam, $5.3 \mathrm{MeV}$ alpha beam and $10.0 \mathrm{MeV}$ carbon beam respectively. A large part of the dose-rate error (70\%) is due to the heterogeneity of the beam.

\section{Survival fraction}

We present here the results of the A549 NSCLC survival curve measured for proton and alpha particles at $1 \mathrm{~Gy} / \mathrm{min}$. The LET values at cell mid-thickness were equal to 25.5 and $102 \mathrm{keV} / \mu \mathrm{m}$. The survival fraction was assessed after clonogenic assays performed 11 days post-irradiation. Results are presented in Figure 5 for the alpha and in Figure 6 for the proton beams. In these figures, one can recognize the characteristic shape obtained with high LET irradiation: the experimental data can be fitted with a linear model. This yields a radiosensitivity parameter $\alpha$ 
$\left(\mathrm{Gy}^{-1}\right)$ equal to1.26 $\pm 0.03 \mathrm{~Gy}^{-1}$ and $2.36 \pm 0.08 \mathrm{~Gy}^{-1}$ for proton and alpha particles respectively. This parameter computes the characteristic intrinsic radiosensitivity of the cell type and the direct mortality of the ionizing particle. We also used these values to calculate the inactivation cross section of A549 NSCLC when exposed to proton and alpha particles according to the equation:

$\sigma=0.16 \operatorname{LET} \alpha\left(\mu \mathrm{m}^{2}\right)$

This yields $5.1 \mu \mathrm{m}^{2}$ for proton and $38 \mu \mathrm{m}^{2}$ for alpha particles. The cross section increases by a factor of 7.4 when moving from protons to alpha particles, whereas the LET increases by a factor of 4. In Figure 7, we compare the results presented by Bromley et al. [26] for the same cell line with X-ray irradiation to the results of our study. Bromley et al. obtained the characteristic shape for survival fraction due to low ionizing radiation with radiosensitivity parameters equal to $0.2432 \mathrm{~Gy}^{-1}$ and $0.0257 \mathrm{~Gy}^{-2}$ for $\alpha$ and $\beta$ respectively. The survival fraction at $2 \mathrm{~Gy}$ is equal to 0.55 for $\mathrm{x}$-rays and to $0.057 \pm 0.019$ and $0.0068 \pm 0.0018$ for proton and alpha particles respectively. The relative biological effectiveness (RBE) for a survival fraction of 0.10 is estimated at 3.2 for the proton beam and 5.9 for alpha beam. In their work, Scampoli et al. obtained similar results for Chinese hamster lung cells V79 [22]. They calculated a RBE equal to 4.7 for $34 \mathrm{keV} / \mu \mathrm{m}$ proton beam and 6.3 for $107 \mathrm{keV} / \mu \mathrm{m}$ alpha particles.

\section{Conclusion}

The in vitro irradiation station developed in our lab allows the irradiation of adherent cells with proton, alpha and carbon broad beams. The dose-rate error has been evaluated and has not exceeded $7 \%$ for all three types of beam. This system allows us to irradiate 100,000 cells simultaneously, and can be used to perform radiobiological experiments like clonogenic assays, immunofluorescence stainings, RNA extraction for gene expression study, morphological 
assessment, and more. In this paper, we present the result of the survival fraction of A549 NSCLC irradiated with proton and alpha beams at $1 \mathrm{~Gy} / \mathrm{min}$. For both irradiations the LET with the maximum RBE was chosen $(25$ and $100 \mathrm{keV} / \mu \mathrm{m})$. The radiosensitivity parameter was

estimated to $1.26 \pm 0.03 \mathrm{~Gy}^{-1}$ and $2.36 \pm 0.08 \mathrm{~Gy}^{-1}$ for proton and alpha particles respectively. The inactivation cross section was also calculated, and this highlighted the higher cell sensitivity when working with alpha particles. The RBE was also derived from X-rays value of the literature and is 3.2 and 5.9 for protons and alpha particles respectively.

\section{Acknowledgements}

We would like to thank Y. Morciaux, A. Nonet and L. Lambotte for their technical support. A.-C. Heuskin is supported by the Belgian Found for Scientific Research (F.R.S. - FNRS). H. Riquier is recipient of a Télévie fellowship. 


\section{$\underline{\text { References }}$}

[1] R.R. Wilson, Radiological use of fast protons, Radiology, 47 (1946) 487-491.

[2] U. Amaldi, Nuclear physics applications in diagnostics and cancer therapy, Nuclear Physics A, 751 (2005) 409c-428c.

[3] E.A. Blakely, New measurements for hadrontherapy and space radiation: biology, Phys Med, 17 Suppl 1 (2001) 50-58.

[4] G. Chuanling, Studies of advantages of heavy ions in radiotherapy compared with gammarays, Nuclear Instruments \& Methods in Physics Research Section B-Beam Interactions with Materials and Atoms, 259 (2007) 997-1003.

[5] Y. Furusawa, M. Aoki, M. Durante, Simultaneous exposure of mammalian cells to heavy ions and X-rays, Adv Space Res, 30 (2002) 877-884.

[6] C.R. Geard, C.Y. Chen, Micronuclei and clonogenicity following low- and high-dose-rate gamma irradiation of normal human fibroblasts, Radiation research, 124 (1990) S56-61.

[7] K.M. Prise, G. Schettino, C. Shao, V. Stewart, L. Wu, N. Zyuzikov, M. Folkard, B.D. Michael, Studies of bystander responses with the GCI microbeams, Radiation research, 161 (2004) 118-119.

[8] H. Zhou, G. Randers-Pehrson, E.J. Hall, D.J. Brenner, C. Geard, T.K. Hei, Interaction of radiation-induced adaptive response and bystander mutagenesis in mammalian cells, Radiation research, 161 (2004) 115-115.

[9] P. Barberet, A. Balana, S. Incerti, C. Michelet-Habchi, P. Moretto, T. Pouthier, Development of a focused charged particle microbeam for the irradiation of individual cells, Review of Scientific Instruments, 76 (2005) -.

[10] M. Cholewa, B.E. Fischer, M. Heiss, Preparatory experiments for a single ion hit facility at GSI, Nuclear Instruments \& Methods in Physics Research Section B-Beam Interactions with Materials and Atoms, 210 (2003) 296-301.

[11] M. Folkard, B. Vojnovic, K.M. Prise, A.G. Bowey, R.J. Locke, G. Schettino, B.D. Michael, A charged-particle microbeam: I. Development of an experimental system for targeting cells individually with counted particles, International journal of radiation biology, 72 (1997) 375-385. [12] K.D. Greif, H.J. Brede, D. Frankenberg, U. Giesen, The PTB single ion microbeam for irradiation of living cells, Nuclear Instruments \& Methods in Physics Research Section B-Beam Interactions with Materials and Atoms, 217 (2004) 505-512.

[13] M. Oikawa, T. Kamiya, M. Fukuda, S. Okumura, H. Inoue, S. Masuno, S. Umemiya, Y. Oshiyama, Y. Taira, Design of a focusing high-energy heavy ion microbeam system at the JAERI AVF cyclotron, Nuclear Instruments \& Methods in Physics Research Section B-Beam Interactions with Materials and Atoms, 210 (2003) 54-58.

[14] G. Randers-Pehrson, C.R. Geard, G. Johnson, C.D. Elliston, D.J. Brenner, The Columbia University single-ion microbeam, Radiation research, 156 (2001) 210-214.

[15] T. Reinert, A. Fiedler, J. Skopek, J. Tanner, J. Vogt, T. Butz, Single ion bombardment of living cells at LIPSION, Nuclear Instruments \& Methods in Physics Research Section B-Beam Interactions with Materials and Atoms, 219-20 (2004) 77-81.

[16] H. Yamaguchi, Y. Sato, H. Imaseki, N. Yasuda, T. Hamano, Y. Furusawa, M. Suzuki, T. Ishikawa, T. Mori, K. Matsumoto, T. Konishi, M. Yukawa, F. Soga, Single particle irradiation 
system to cell (SPICE) at NIRS, Nuclear Instruments \& Methods in Physics Research Section BBeam Interactions with Materials and Atoms, 210 (2003) 292-295.

[17] I. Bailly, C. Champion, P. Massiot, P. Savarin, J.L. Poncy, S. Crespin, G. Alloy, V. Jacob, E. Petibon, Development of an experimental system for biological studies: Scintillation and solidtrack detectors as dose monitors, Nuclear Instruments \& Methods in Physics Research Section BBeam Interactions with Materials and Atoms, 187 (2002) 137-148.

[18] M. Belli, R. Cherubini, G. Galeazzi, S. Mazzucato, G. Moschini, O. Sapora, G. Simone, M.A. Tabocchini, Proton Irradiation Facility for Radiobiological Studies at a $7 \mathrm{mv}$ Vandegraaff Accelerator, Nuclear Instruments \& Methods in Physics Research Section a-Accelerators Spectrometers Detectors and Associated Equipment, 256 (1987) 576-580.

[19] J. Besserer, J. de Boer, M. Dellert, C. Gahn, M. Moosburger, P. Pemler, P. Quicken, L. Distel, H. Schussler, An irradiation facility with a vertical beam for radiobiological studies, Nuclear Instruments \& Methods in Physics Research Section a-Accelerators Spectrometers Detectors and Associated Equipment, 430 (1999) 154-160.

[20] M. Folkard, K.M. Prise, B. Vojnovic, H.C. Newman, M.J. Roper, B.D. Michael, Inactivation of V79 cells by low-energy protons, deuterons and helium-3 ions, International journal of radiation biology, 69 (1996) 729-738.

[21] C.R. Geard, G. Jenkins-Baker, S.A. Marino, B. Ponnaiya, Novel approaches with track segment alpha particles and cell co-cultures in studies of bystander effects, Radiation protection dosimetry, 99 (2002) 233-236.

[22] P. Scampoli, M. Casale, M. Durante, G. Grossi, M. Pugliese, G. Gialanella, Low-energy light ion irradiation beam-line for radiobiological studies, Nuclear Instruments \& Methods in Physics Research Section B-Beam Interactions with Materials and Atoms, 174 (2001) 337-343.

[23] A.C. Wera, K. Donato, C. Michlels, Y. Jongen, S. Lucas, Preliminary results of proton beam characterization for a facility of broad beam in vitro cell irradiation, Nuclear Instruments \& Methods in Physics Research Section B-Beam Interactions with Materials and Atoms, 266 (2008) 2122-2124.

[24] J. Demarche, G. Terwagne, Precise measurement of the differential cross section from the O-16(alpha,alpha)O-16 elastic reaction at 165 degrees and 170 degrees between 2.4 and 6.0 MeV, Journal of Applied Physics, 100 (2006) -.

[25] J.F. Ziegler, J.P. Biersack, U. Littmark, The stopping and range of ions in solids, Pergamon Press, 1985.

[26] R. Bromley, L. Oliver, R. Davey, R. Harvie, C. Baldock, Predicting the clonogenic survival of A549 cells after modulated x-ray irradiation using the linear quadratic model, Physics in medicine and biology, 54 (2009) 187-206. 


\section{Figures}

figure 1: In vitro irradiation station for a broad beam. (1) ${ }^{+} \mathrm{H},{ }^{2+} \mathrm{He},{ }^{4+} \mathrm{C}$ broad beam; (2) vacuum chamber; (3) pumping system; (4) BC400 scintillator + CCD camera fixed on a pneumatic jack; (5) PIPS detector placed on the left side of the beam for the dose-rate monitoring; (6) irradiation head; (7) movable PIPS detector; (8) XY motor. Detail of the irradiation head: (a) stainless steel cylinder; (b) $8 \mu \mathrm{m}$ Kapton foil; (c) plastic cap.

figure 2: Proton energy spectra in vacuum $(\mathrm{E}=1.2 \mathrm{MeV}, \mathrm{FWHM}=7 \mathrm{keV})$ and after the Kapton foil $(\mathrm{E}=952$ $\mathrm{keV}, \mathrm{FWHM}=25 \mathrm{keV}$ ) as measured with calibrated PIPS detector.

figure 3: 5.3 MeV alpha beam stability over time. The dose-rate was set to $1 \mathrm{~Gy} / \mathrm{min}$ and the calculated standard deviation was equal to $2.8 \%$.

figure 4: 5.3 MeV alpha beam map. The incident flux was measured every millimeter, line by line, with a PIPS detector. The dose-rate was calculated using the LET and the flux.

figure 5: A549 cells survival fraction 11 days after irradiation at $1 \mathrm{~Gy} / \mathrm{min}$ with a $5.3 \mathrm{MeV}$ alpha broad beam. The experimental data were obtained with conventional colony forming assays. Experimental data (filled triangle) and linear model fit (straight line) are displayed. Results are expressed as means \pm S.D. ( $n=3$ ).

figure 6: A549 cells survival fraction 11 days after irradiation at $1 \mathrm{~Gy} / \mathrm{min}$ with $1.2 \mathrm{MeV}$ proton broad beam. The experimental data were obtained with conventional colony forming assays. Experimental data (filled square) and linear model fit (straight line) are displayed. Results are expressed as means \pm S.D. (n=3).

figure 7: Comparison of A549 lung cancer cells survival fraction as obtained by different studies. Filled triangle: this study with alpha broad beam; filled square: this study with proton broad beam; star: Bromley et al. (2009) with $x$-rays. 


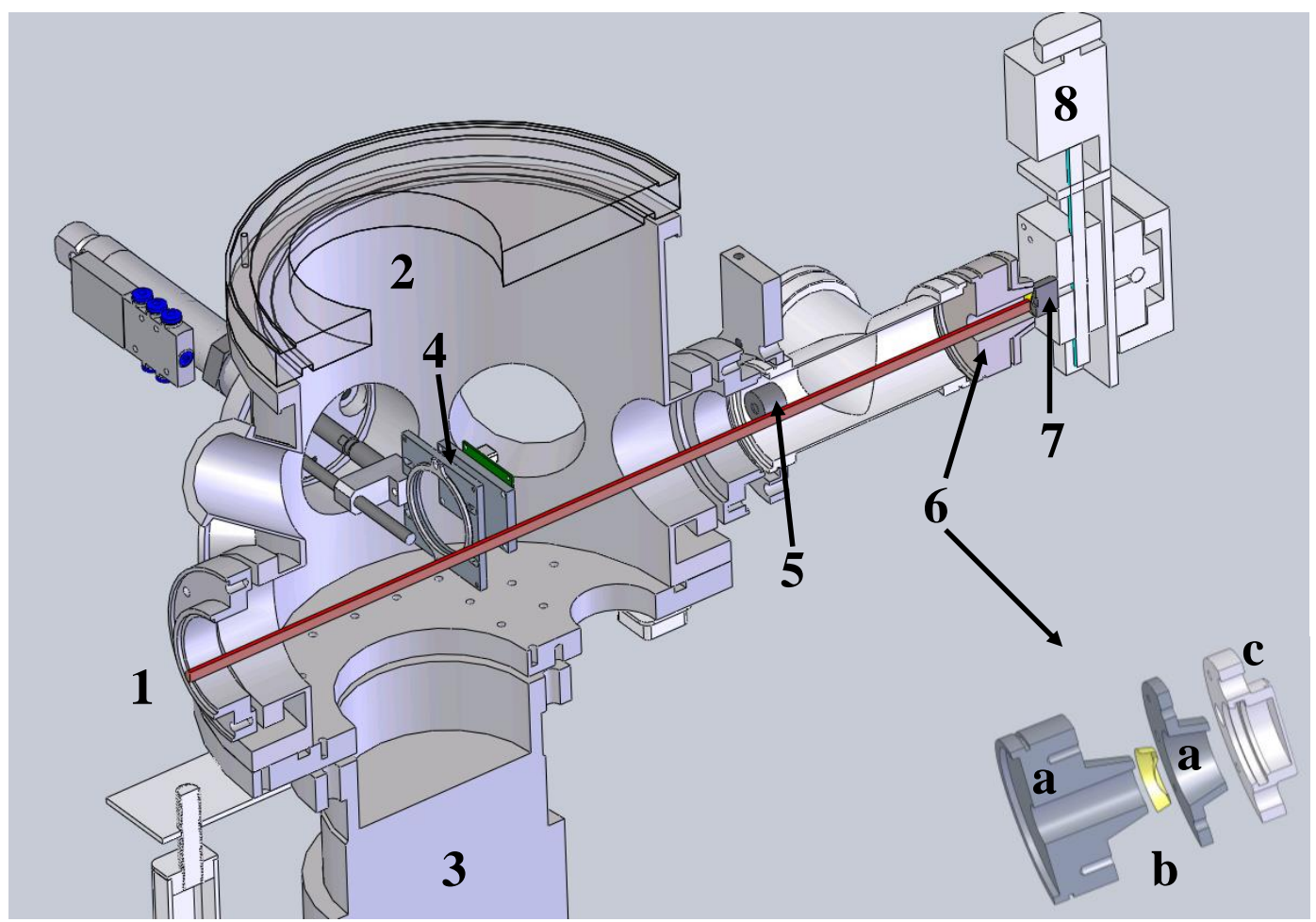

Figure 1 


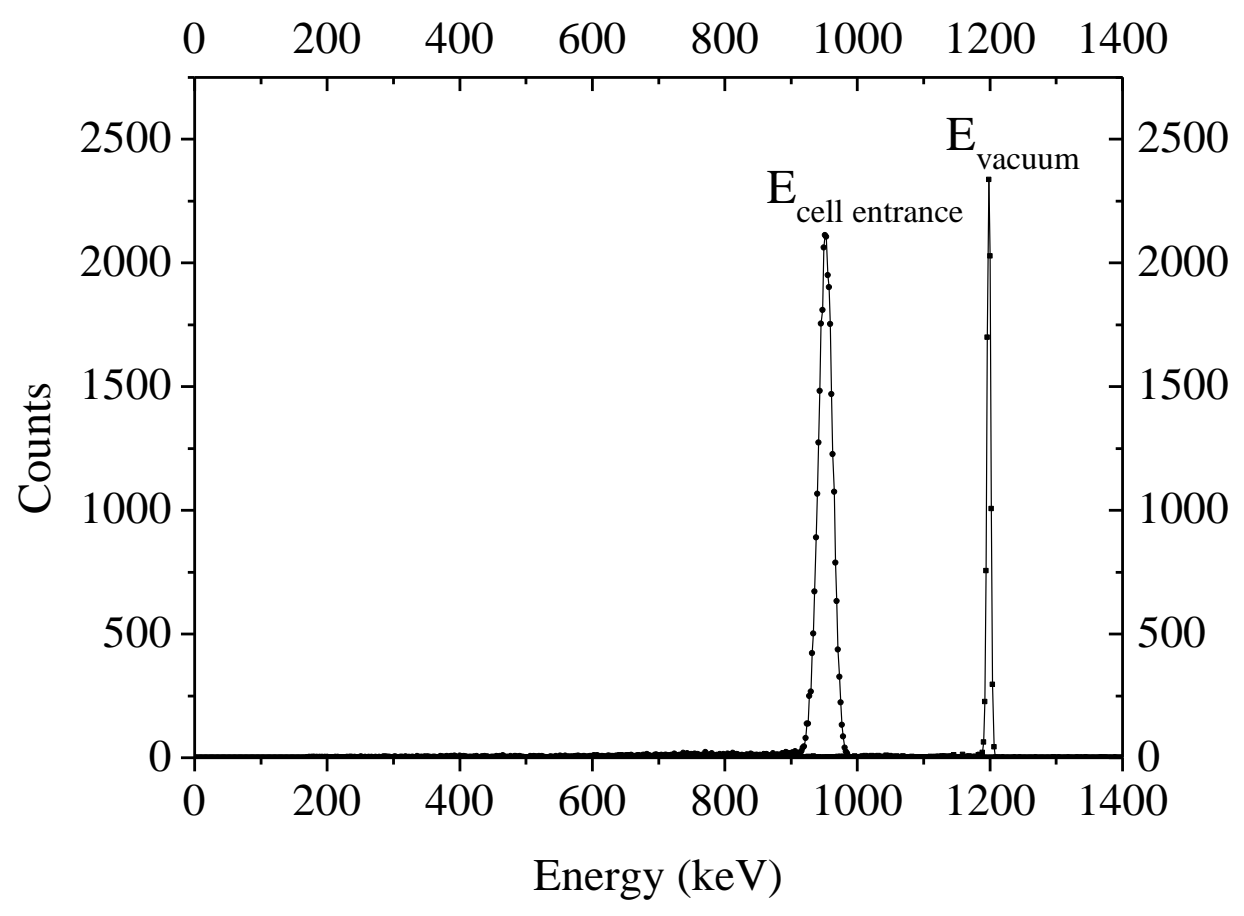

Figure 2 


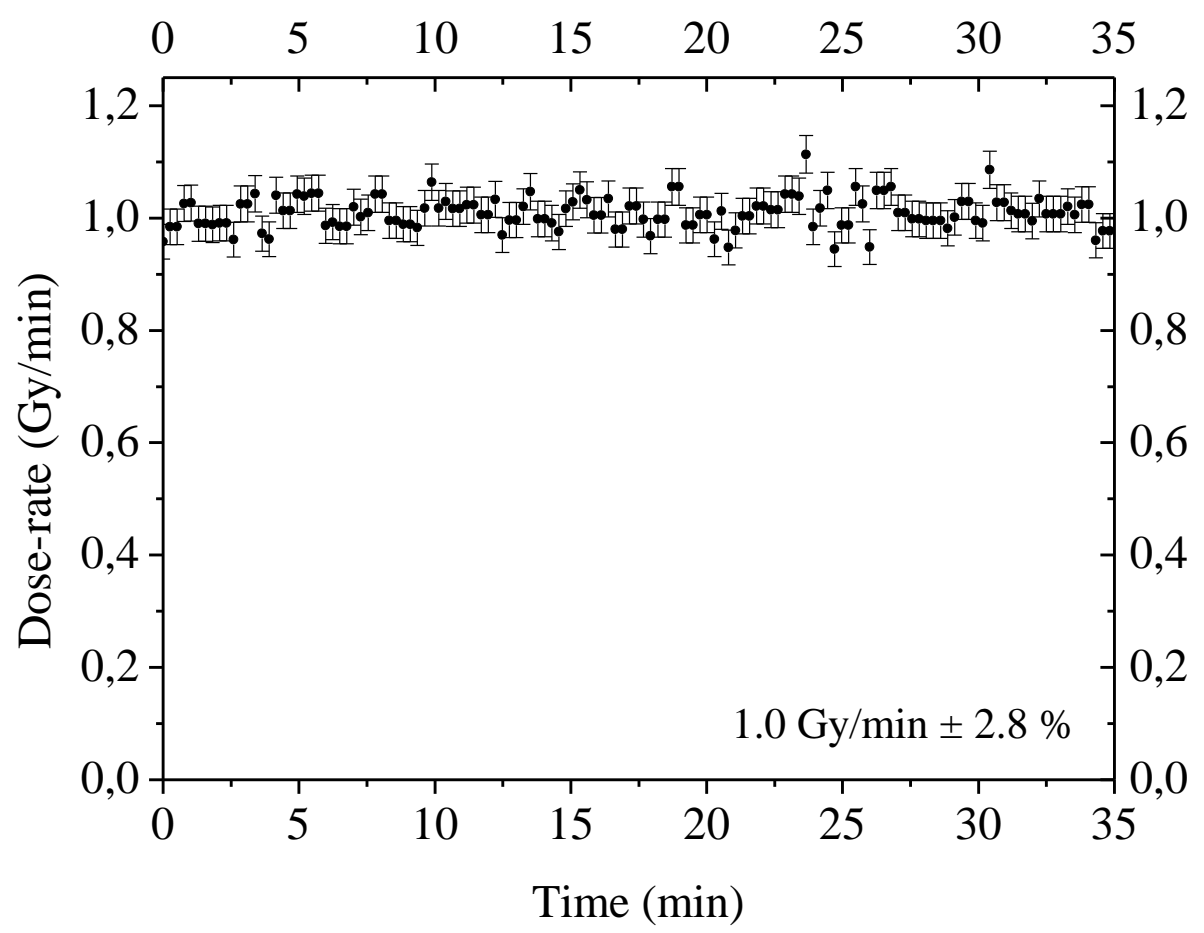

Figure 3 


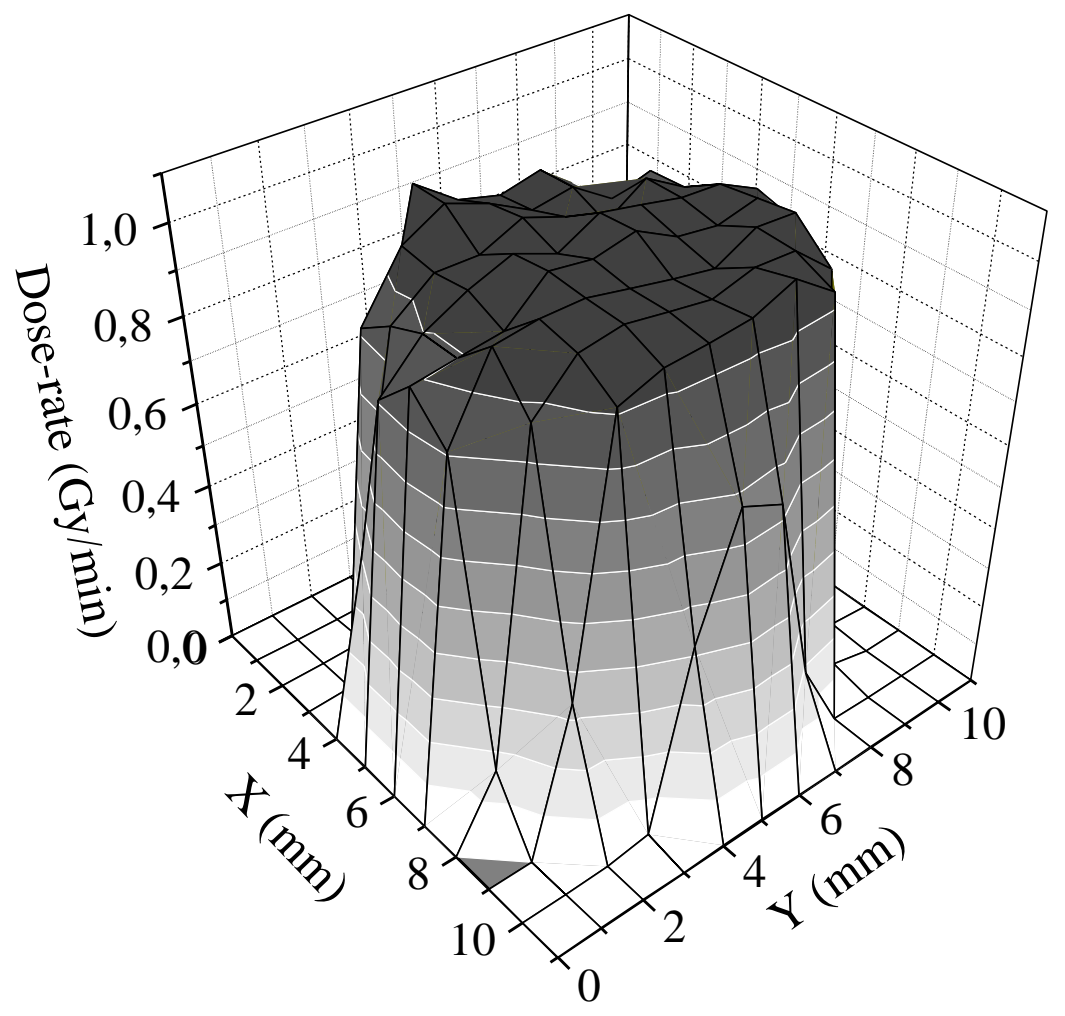

Figure 4 


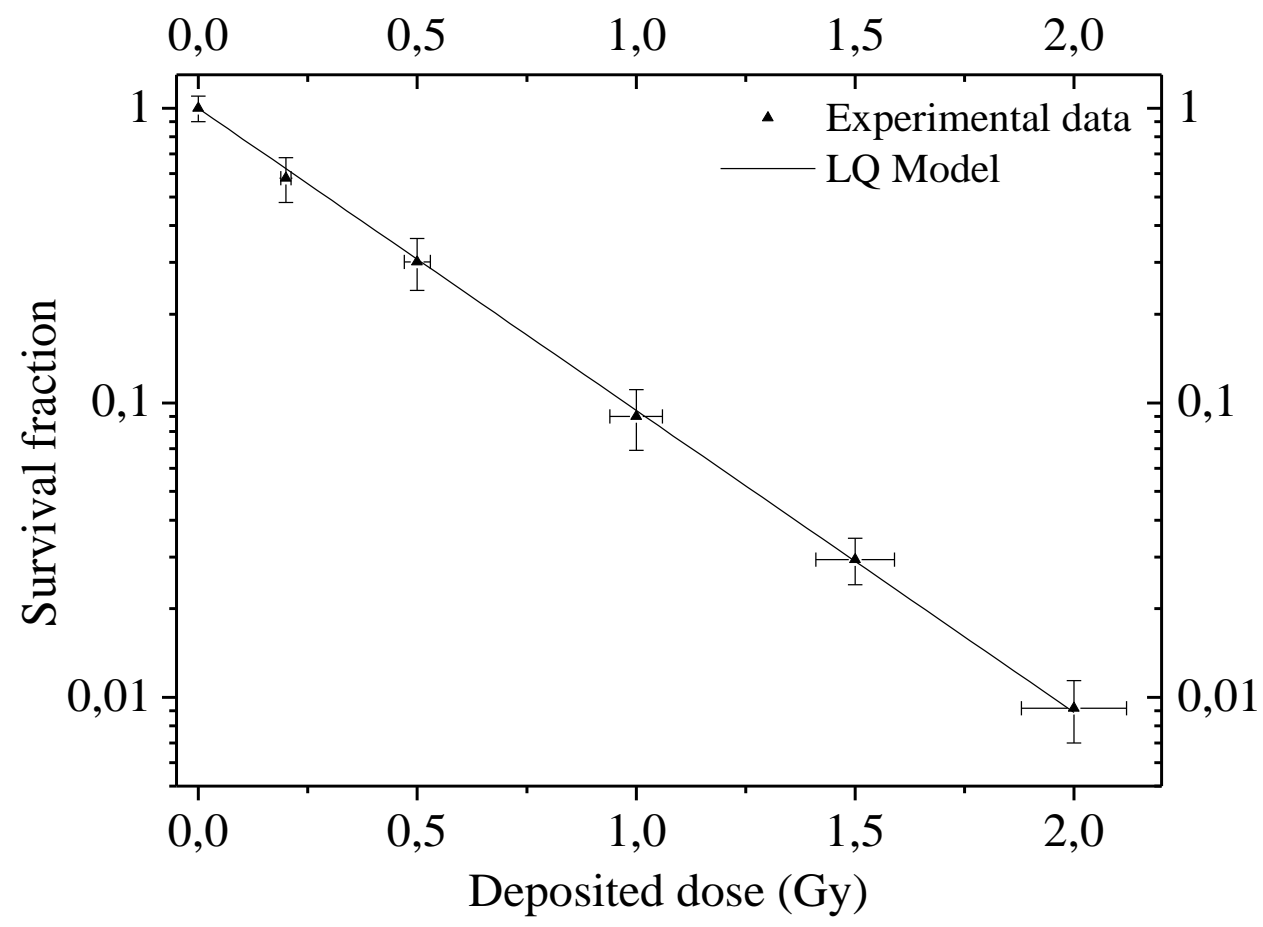

Figure 5 


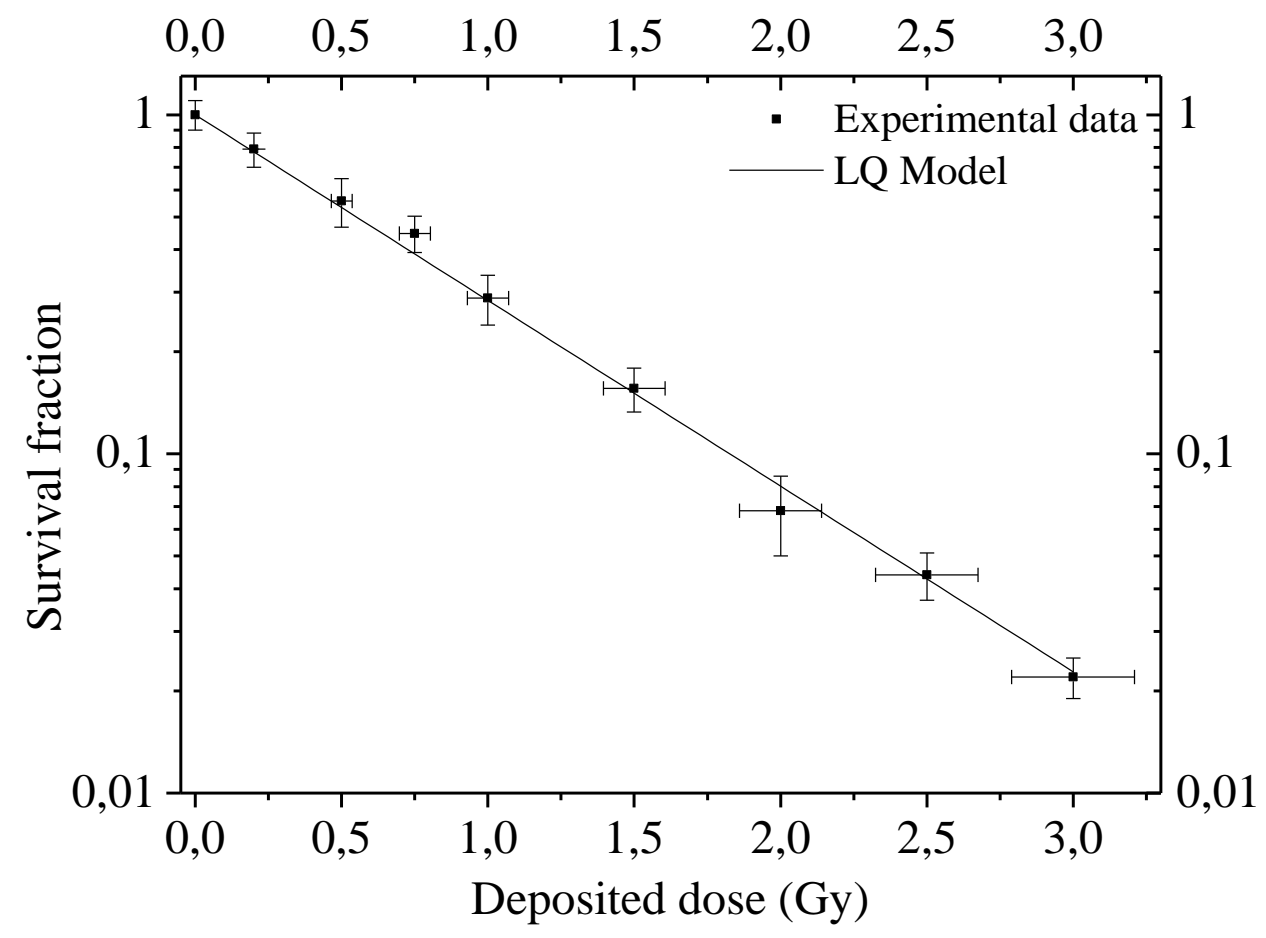

Figure 6 


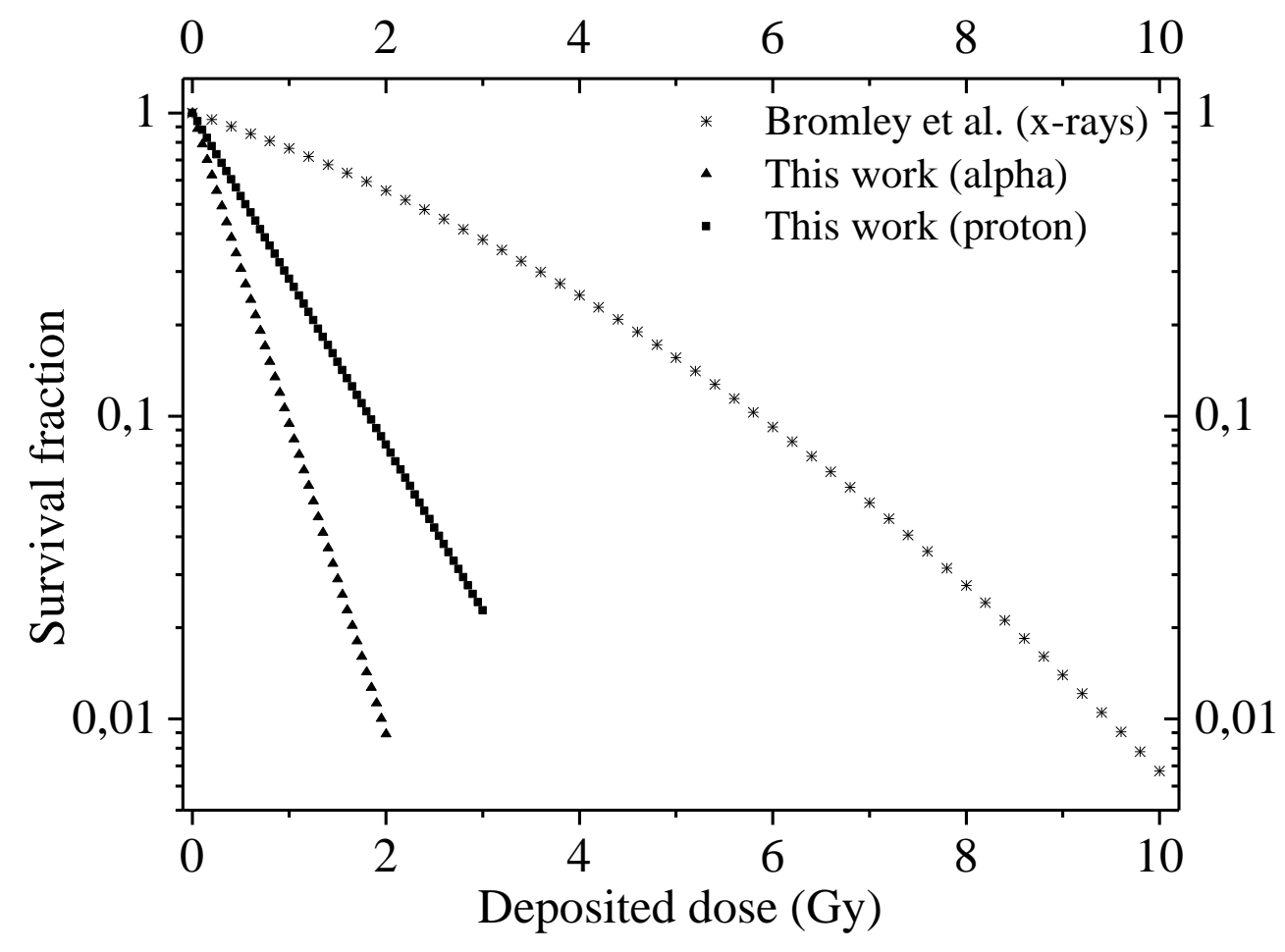

Figure 7 